\title{
Die Zukunft der Mitbestimmung in den Niederlanden - Vier Szenarios
}

Betriebsratsarbeit ist in den Niederlanden heute mit differenzierter Organisationssteuerung in den Unternehmen, dezentralisierten Tarifverhandlungen und zunehmend heterogenen Belegschaften konfrontiert. Angesichts dieser Herausforderungen stellt sich die Frage, wie die verschiedenen Einflussfaktoren auf die Betriebsräte wirken - und das wirft letztlich die Frage auf, nach der Zukunft der Mitbestimmung. Ein gemischtes Team von Praktikern und Wissenschaftlern hat vier realistische Zukunftsszenarios der Betriebsratsarbeit entwickelt: Mitbestimmung in der Organisationsentwicklung, Mitbestimmung in Netzwerken, Betriebsrat als Partner der Gewerkschaften und Unternehmensgewerkschaften. Die dargestellten Entwicklungen könnten - durchaus parallel - die Mitbestimmungslandschaft der Zukunft prägen. ${ }^{1}$

\section{Einleitung}

Ausgangspunkt unserer Studie ist die Frage, wie sich die Mitbestimmung im Allgemeinen und die Betriebsräte im Besonderen an die Realitäten der heutigen Wirtschafts- und Gesellschaftsordnung anpassen. Hierzu zählen die Dezentralisierung und Fragmentierung der industriellen Beziehungen, die ungleichzeitige Internationalisierung von Märkten und marktkorrigierenden Institutionen, der Wandel der Corporate Governance, neue Trends bei Personalmanagement und Organisationsentwicklung, die Flexibilisierung des Arbeitsmarkts und Änderungen des rechtlichen Rahmens mit erweiterter Selbstregulierung. Die möglichen Anpassungsweisen betrieblicher Mitbestimmung wurden vom Forscherteam ${ }^{2}$ als alternative Szenarios entwickelt.

Die Szenariotechnik ist immer dann ein hilfreiches Instrument, wenn politische Entscheidungen getroffen werden müssen. Politische Entscheidungen und ihre Auswirkungen lassen sich mit Szenarios analysieren, die historische Trends fortschreiben und so mögliche Konsequenzen ausleuchten. Ein Szenario ist, so lautet die Definition, die Beschreibung einer möglichen Situation in der Zukunft, die zusammen mit einem widerspruchsfreien Bündel weiterer Szenarios den Raum möglicher zukünftiger Entwicklungen skizziert. Methodisch beruht die Studie auf den Arbeiten von Schwartz (1991) und Becker (1994).

Zur Definition des Szenariofelds dienen einige Aspekte der niederländischen Mitbestimmung (Abschnitt2), auf die sechs
Einflussfaktoren wirken (Abschnitt 3). Aus dem Szenariofeld und den Wirkungen der Einflussfaktoren lässt sich eine Szenariologik entwickeln, die in unterschiedliche Szenarios übersetzt und an konkreten Beispielen illustriert wird (Abschnitt 4). Die Schlussfolgerungen enthalten erste Überlegungen zur wahrscheinlichen Verbreitung der unterschiedlichen Szenarios in der mittleren Zukunft (Abschnitt 5).

Die Daten- und Informationsbeschaffung beruhte in erster Linie auf Literaturrecherchen und Sekundärforschung der Teammitglieder. Für die kreativen Arbeitsabschnitte der Analyse, für die Entwicklung und Gestaltung der Szenarios wurden Teamsitzungen und Workshops organisiert. Um eine externe Kontrolle zu gewährleisten, wurde ein Expertentreffen durchgeführt. Das Ergebnis der Arbeitsgruppe bestand in vier sich gegenseitig nicht ausschließenden Szenarios (Van het Kaar/Smit 2007). Nach Abschluss der Studie wurden vier Betriebsräte aus Betrieben, die jeweils eines der Szenarios repräsentierten, ausgewählt und zu einem von vier Workshops eingeladen, um das jeweilige Szenario einem Praxistest zu unterziehen (Smit/Vonk, im Erscheinen).

\section{Mitbestimmung in den Niederlanden}

In den Niederlanden sind alle Unternehmen mit mindestens 50 Beschäftigten verpflichtet, einen Betriebsrat einzusetzen. Das niederländische Betriebsratsgesetz weist ihm eine paradoxe Doppelrolle zu. Der Betriebsrat wird „im Interesse des guten
Funktionierens des Unternehmens in all seinen Zielsetzungen“ und zugleich „zum Zweck der Vertretung der in dem Unternehmen beschäftigten Personen" eingesetzt. Er verfügt über drei wesentliche Befugnisse:

Erstens hat er Beratungsrechte, denen zufolge der Unternehmer den Betriebsrat vor allen wichtigen strategischen, wirtschaftlichen und organisatorischen Entscheidungen konsultieren muss. Wenn die endgültige Entscheidung des Unterneh-

\footnotetext{
1 Im Auftrag des niederländischen Ministeriums für Soziales und Beschäftigung führten das Hugo Sinzheimer Institut der Universität Amsterdam und die Organisationsberatung Basis \& Beleid 2006 eine Studie über die Zukunft der Betriebsratsarbeit in den Niederlanden durch. Dieser Beitrag fasst die Studie zusammen und gibt einen knappen Überblick über die Ergebnisse von Workshops mit Praktikern, in denen die aus der Studie entwickelten Szenarios durchgespielt wurden.

2 Wout Buitelaar, Marc van der Meer, Jan Popma, Frank Tros, Robbert van het Kaar (Universität Amsterdam) und Robin Colard, Evert Smit (Basis \& Beleid).
}

Evert Smit, Soziologe, Consultant bei der
Organisationsberatung Basis \& Beleid,
Utrecht. Arbeitsschwerpunkte: Arbeitsbezie-
hungen, Betriebsratsarbeit, Tarifpolitik.
e-mail: Evertsmit@basisenbeleid.nl
Robbert van het Kaar, Dr., Hugo Sinzheimer
Institut, Universität Amsterdam. Arbeits-
schwerpunkte: Mitbestimmung in Europa,
Corporate Governance.
e-mail: R.H.vanhetKaar@uva.nl

Übersetzung aus dem Englischen von Stefan Matzig 
mers nicht dem Vorschlag des Betriebsrats entspricht, kann er diese vor der Unternehmenskammer des Berufungsgerichts Amsterdam anfechten. Zwar wird das Gericht die Vorgehensweise nur auf Verfahrensfehler prüfen, doch verleiht das Rechtsmittel dem Beratungsrecht ein recht großes Gewicht (Van het Kaar 2007).

Zweitens muss der Unternehmer vor allen Änderungen der Berufsstatuten und Arbeitsbedingungen die Zustimmung des Betriebsrats einholen, sofern sie nicht in einem Tarifvertrag $(\mathrm{CAO})$ geregelt sind.

Drittens hat der Betriebsrat uneingeschränkt Anspruch auf die rechtzeitige Vorlage aller Informationen und Daten, die er zur Wahrnehmung seiner Aufgaben benötigt. Auch dies ist im Vergleich mit den Rechten der Betriebsräte in anderen europäischen Ländern eine starke Position. Andererseits hat der Betriebsrat bei der Besetzung des Aufsichtsrats lediglich ein Vorschlagsrecht und er verfügt über keine formale Handhabe, auf die Aufsichtsräte multinationaler Gesellschaften Einfluss zu nehmen. Dies ist im Vergleich zu Ländern wie Schweden und Deutschland eine schwächere Position (Van het Kaar 2007).

\section{Einflussfaktoren}

\subsection{SYSTEM INDUSTRIELLER BEZIEHUNGEN UND MITBESTIMMUNG}

Die Frage, wie sich Gewerkschaften in Zukunft entwickeln, ist von entscheidender Bedeutung für die Betriebsratsarbeit. Obwohl sich ihre Mitgliederzahlen erkennbar stabilisieren konnten, ist der gewerkschaftliche Organisationsgrad in den Niederlanden stetig gesunken. Er beträgt derzeit im Durchschnitt 22 \% (Visser 2006). Der Anteil der Gewerkschaftsmitglieder an der Gesamtbelegschaft in den einzelnen Sektoren ist höchst unterschiedlich. In geschützten Sektoren, die nicht oder nur in begrenztem Ausmaß dem internationalen Wettbewerb ausgesetzt sind, findet sich ein überdurchschnittlich hoher gewerkschaftlicher Organisationsgrad, während der Dienstleistungssektor, der im internationalen Wettbewerb steht, stark unterorganisiert ist.

Trotzdem sind Tarifverträge nach wie vor weit verbreitet. Rechnet man die Ver- einbarungen für die Beschäftigten des öffentlichen Dienstes hinzu, so gilt für $82 \%$ der Beschäftigten ein Tarifvertrag (Visser 2006). Gleichzeitig empfindet man die größer werdende Lücke zwischen dem Geltungsbereich der tariflichen Absicherung und dem - rückläufigen - gewerkschaftlichen Organisationsgrad zunehmend als Problem. Erste Risse im Tarifsystem machen sich nun insbesondere in Bereichen mit einem geringen Organisationsgrad bemerkbar. Es erscheint durchaus möglich, dass einzelne Branchentarifverträge in $\mathrm{Zu}-$ kunft nicht mehr erneuert werden (Schilstra/Smit 2004). Wenn das System der Allgemeinverbindlichkeit von Tarifverträgen abgeschafft oder aufgeweicht wird, dürfte dieser Fall zwangsläufig eintreten. An der Notwendigkeit kollektiver Tarifverträge wird sich dadurch zwar nichts ändern, doch werden dann die Betriebsräte gezwungen sein, in diesen Fragen selbst Stellung zu beziehen.

In den industriellen Beziehungen gewinnt die Unternehmensebene zunehmend an Bedeutung. Den Betriebsräten wurden in der jüngeren Vergangenheit immer mehr Aufgaben übertragen, nicht zuletzt die Durchführung des Arbeitszeitgesetzes und des Arbeitsschutzgesetzes. Ferner setzen Arbeitgeber und Gewerkschaften bei der Regelung der Arbeitsbedingungen verstärkt auf Unternehmensvereinbarungen. Einerseits nehmen derartige Vereinbarungen die Form eines Wahltarifvertrags an (Tarifvertrag à la carte), andererseits lässt die geschichtete Vertragsstruktur auf Unternehmensebene Raum für Interpretationen. Wenn die Arbeitsbedingungen in koordinierter Form fortentwickelt werden sollen, wird der Betriebsrat letztendlich in die Rolle des Verhandlungspartners und Mitgestalters der Sozialpolitik schlüpfen oder die Kontrolle der von den Tarifparteien vereinbarten Verfahren übernehmen müssen. Der Betriebsrat könnte in Unternehmen mit geringem gewerkschaftlichen Organisationsgrad sogar zur Gewerkschaftsalternative aufgewertet werden.

Die Sicherstellung der Tarifverhandlungen auf Unternehmensebene wirft einige strittige Fragen auf: Die Beziehungen zwischen Betriebsrat und Gewerkschaften werden wieder auf die Tagesordnung kommen. Zudem müssen Regelungen gefunden werden, wie die Vereinbarungen des Betriebsrats in die individuellen Arbeitsverträge eingehen.

\subsection{INTERNATIONALISIERUNC UND MITBESTIMMUNG}

Der Prozess der wirtschaftlichen Globalisierung führte in den letzten zwei Jahrzehnten einerseits zur Öffnung internationaler Märkte, zu erhöhter Mobilität des internationalen Kapitals und zur internationalen Verbreitung technischer Anwendungen, erfolgreicher Vorbilder und best practices. Andererseits ist festzustellen, dass zwischen den Tarifsystemen und Mitbestimmungsregeln in den westlichen Ländern nach wie vor große Unterschiede bestehen.

Der Ausbau der Marktmechanismen ist in Europa weiter fortgeschritten als die soziale Integration. Gleichzeitig ist der Einfluss der europäischen Politik auf die nationale Ausgestaltung der Arbeitsbedingungen recht begrenzt geblieben. So sind die Bemühungen der internationalen $\mathrm{Me}$ tallgewerkschaften zur Koordinierung der Tarifpolitik nicht auf fruchtbaren Boden gefallen und die gemeinsame Koordinierung der Arbeitsbedingungen in Deutschland, den Niederlanden und Belgien vorerst gescheitert. Auf internationaler Ebene sieht es in Bezug auf handlungsfähige Akteure bzw. belastbare Koordinationsverfahren kaum besser aus. Letztlich führen diese Defizite dazu, dass die Mitbestimmung in den niederländischen Unternehmen und Institutionen dem direkten und indirekten Einfluss der wirtschaftlichen Internationalisierung nahezu ungeschützt ausgesetzt ist.

\subsection{UNTERNEHMENSFÜHRUNG UND MITBESTIMMUNG}

Ein wesentlicher Grundsatz des Betriebsratsgesetzes besteht darin, dass alle Mitwirkungsrechte an die Entscheidungsprozesse der Unternehmensleitung gebunden sind. Veränderungen im Bereich der Corporate Governance betreffen jedoch die Bedingungen der Entscheidungsfindung in Unternehmen. So weiten die Aktionäre ihre Einflussnahme auf das Management aus, während auf der anderen Seite der Spielraum des Aufsichtsrats einschränkt wird und somit auch der Raum, der für die Mitbestimmung zur Verfügung steht. Es ist nicht zu übersehen, dass in verschiedenen Ländern die Arbeitnehmervertretung in Unternehmensorganen wieder einmal unter Druck geraten ist.

Solange im Bereich der halbstaatlichen und staatlichen Unternehmen weiterhin alternative Modelle der Corporate Gover- 
nance bestehen, dürfte die geschilderte Aufwertung des Aktionärseinflusses die Mitbestimmung nicht vollständig prägen. Außerdem verläuft die Entwicklung der Unternehmensmitbestimmung nicht geradlinig. Der zunehmende Aktionärsaktivismus setzt die bestehenden Formen der Unternehmensmitbestimmung zwar stark unter Druck, dennoch erleben diese auf EU-Ebene aus niederländischer Sicht gerade einen zweiten Frühling (Europäische Gesellschaft SE, Europäische Genossenschaft SCE, grenzüberschreitende Fusionen).

\subsection{PERSONALMANAGEMENT, ORGANISATIONSENTWICKLUNG UND MITBESTIMMUNG}

Die Personalpolitik der Unternehmen hat sich in den letzten Jahrzehnten zum sogenannten Personalmanagement (Human Resource Management) weiterentwickelt. Aufgabe des Personalmanagements ist es, dem Unternehmen durch den strategischen Einsatz engagierter und kompetenter Arbeitnehmer einen Wettbewerbsvorteil zu verschaffen. Kerngedanke dieser Neuorientierung im Personalmanagement ist es, Formen der direkten Anweisung und Kontrolle der Beschäftigten durch eine Strategie zu ersetzen, die auf das persönliche Engagement der Beschäftigten für die jeweiligen Aufgabenbereiche setzt und ihre Identifikation mit dem Unternehmensziel sicherstellt.

Bei der konzeptuellen Ausgestaltung der Organisationsentwicklung nimmt die Idee der lernenden Organisation großen Raum ein. Im Zentrum einer lernenden Organisation steht ihre Weiterentwicklung, wozu auch die laufende Neubeurteilung der Kontrollen und Hierarchien gehört (Nonaka/Takeuchi 1995).

Hier bietet sich der Arbeitnehmervertretung eine exzellente Gelegenheit, neue Formen der Arbeitnehmerpartizipation und Organisationsentwicklung zu erproben. Zusammen mit dem Personalmanagement könnte man auf diese Weise das Fundament für die lernende Organisation der Zukunft legen. Es ist deshalb nicht mehr unvorstellbar, dass die formale Mitbestimmung in Zukunft an Bedeutung verlieren könnte.

\subsection{ARBEITSMARKT-FLEXIBILISIERUNG UND MITBESTIMMUNG}

Die strukturellen Entwicklungen auf dem Arbeitsmarkt schaffen neue Aufgaben für den Betriebsrat, insbesondere dort, wo es um zunehmenden Flexibilitätsbedarf (zahlenmäßig, funktionell, Entlohnung) der Unternehmen geht. Gleichzeitig wünschen sich die Arbeitgeber von ihren Arbeitnehmern eine Form der Loyalität, die dem hohen Grad an Flexibilisierung widerspricht. Insgesamt nimmt jedoch die Vielfalt der individuellen Arbeitsbeziehungen in Bezug auf Vergütung, Befristung, Vermittlungsfähigkeit, Motivation etc. zu. Auch die wachsende Heterogenität der Belegschaften im Hinblick auf Herkunft, Ausbildungsstand, Geschlecht, Alter, (flexible) Vertragsgestaltung und Position auf dem internen und externen Arbeitsmarkt muss in einer repräsentativen Personalvertretung abgebildet werden.

Für Betriebsräte, die sich als kollektive Interessenvertreter verstehen, wird die Situation jedoch schwieriger werden. In ihren Beziehungen orientieren sich Arbeitgeber und Arbeitnehmer nach und nach an einem "neuen psychologischen Vertrag" (Marsden 2004), dem unausgesprochenen Tausch von Loyalität für Arbeitsplatzsicherheit und Karriere. Ein solcher psychologischer Vertrag unterliegt der individuellen Ausgestaltung und hat eine weniger langfristige Bindung zum Ziel. Entsprechend geht der Einfluss der Betriebsräte auf der zentralen Unternehmensebene zurück, während ihre Rolle als Informationsanbieter und Vermittler in Einzelfällen immer relevanter wird.

\section{6 ÄNDERUNGEN DES RECHTLICHEN RAHMENS}

Im Bereich der Gesetzgebung lässt sich beobachten, dass die klassische hoheitliche Steuerung über Ge- und Verbote - teilweise infolge eines neuen Politikverständnisses - durch Selbstregulierung verschiedener Prägungen ersetzt wird (De Schutter/Deakin 2005). Eine solche Selbstregulierung braucht einen gesetzlich definierten Raum, in dem die Unternehmen ihre Sorgfaltspflichten unter Beweis stellen und mit den Interessengruppen, insbesondere den Arbeitnehmervertretern, in einen Dialog treten können.

In der gegenwärtigen Situation in den Niederlanden hat dies die Form der „Dreiviertel-Regelung" angenommen: Arbeitgeber und Gewerkschaften dürfen dabei die rechtlichen Normen in ihren Tarifverträgen innerhalb bestimmter Grenzen auslegen oder sogar von ihnen abweichen. Im- mer häufiger kommt es daneben zum Abschluss von Vereinbarungen auf Betriebsebene. Vertragsgegenstände sind hier in erster Linie die gesetzlichen Arbeitsbedingungen, die Arbeitszeiten und die Arbeitssicherheit.

Es ist anzunehmen, dass dieses Verfahren innerhalb des Arbeitsrechts Schule machen wird. Der Gesetzgeber legt die Rahmenbedingungen fest, und die Tarifparteien gestalten sie auf Branchen- oder Unternehmensebene aus. Diese Form der „regulierten Selbstregulierung “ könnte gröBere Belastungen für die Betriebsräte mit sich bringen. Dies schließt eine wirkungsvolle Mitbestimmung nicht aus, wenn jedoch die Träger der Mitbestimmung nicht adäquat ausgestattet sind und keine Gegenmacht entfalten können, könnte die Selbstregulierung zur Mitbestimmungskarikatur verkommen.

\subsection{DIE DREI HAUPTTRENDS}

Die Analyse der Kontextfaktoren lässt sich zu den folgenden drei Trends verdichten:

\section{DIFFERENZIERUNG DER ORGANISATIONSSTEUERUNG}

Einerseits wird das Zentrum der Kontrolle, also letztlich der Ansprechpartner der Arbeitnehmervertretung, außerhalb der Organisation angesiedelt. Die Gründe hierfür liegen in der Internationalisierung (Kontrolle wird auf supranationaler Ebene ausgeübt) und der Schaffung von Organisationen mit einer Netzwerkstruktur (Arbeitnehmervertreter sind mit Entscheidungen konfrontiert, die an anderen Stellen der Struktur getroffen werden). Andererseits werden innerhalb der Organisationen hierarchische Strukturen tendenziell abgebaut.

Für die Mitbestimmung bedeutet dies, dass der Zugriff auf die strategischen Entscheidungsprozesse verbessert werden muss, obwohl die Möglichkeiten hierfür zu schwinden scheinen. Spiegelbildlich dazu verringert der Abbau der unternehmensinternen Hierarchien die Machtdistanz zwischen dem (lokalen) Management und den Arbeitnehmern. Hier bieten sich zunehmend Möglichkeiten für eine Arbeitnehmerbeteiligung im Personalmanagement und in der Organisationsentwicklung. 


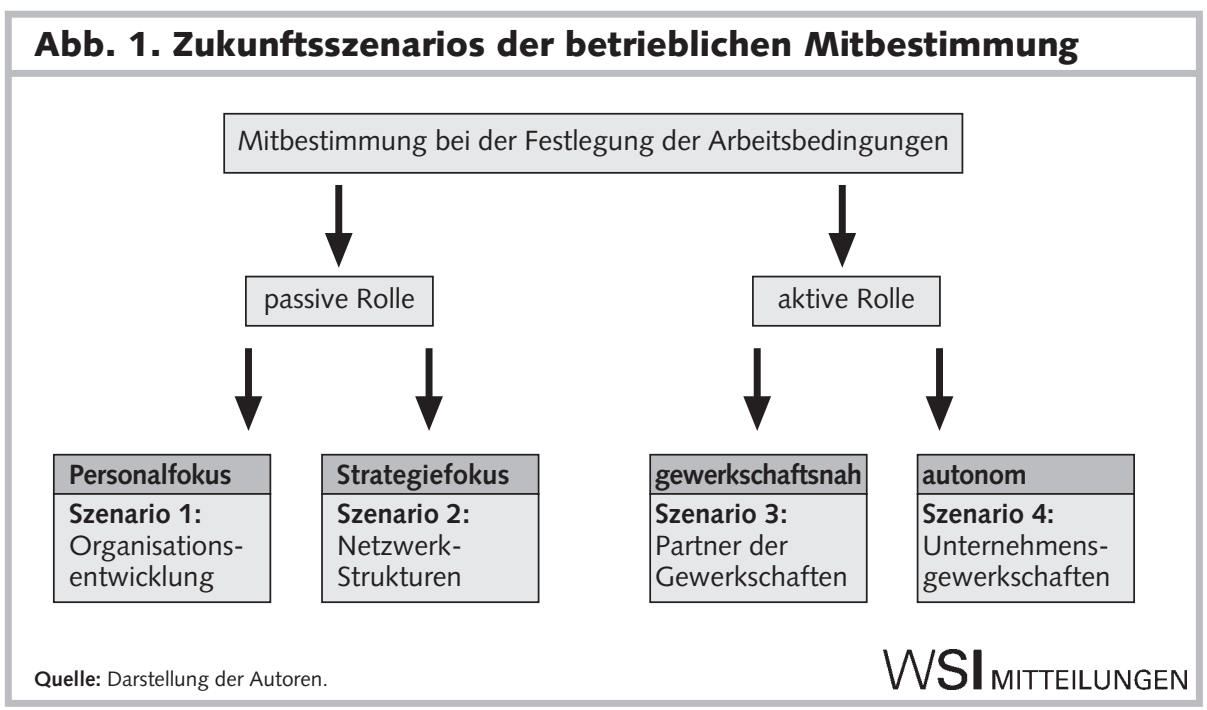

\section{DEZENTRALISIERUNG DER TARIFVERHANDLUNGEN}

Man kann davon ausgehen, dass die Verlagerung der Tarifverhandlungen von der Branchen- auf die Unternehmensebene und von der Unternehmensebene in die Abteilungen und Teams auf die Einführung und Ausweitung der individuellen Wahlmöglichkeiten in den Tarifverträgen folgen wird. Mit einer rezentralisierten Entwicklung der Arbeitsbedingungen könnte dies, wie die neuen Branchentarifverträge zeigen, in gewissem Umfang ausgeglichen werden. Die Betriebsräte werden trotzdem auch in diesem Bereich weitere Aufgaben wahrnehmen und sich festlegen müssen, welche Rolle sie in den Arbeitsbeziehungen spielen wollen. Hier scheint es unausweichlich, die Beziehungen und Arbeitsteilungen zwischen Betriebsräten und Gewerkschaften künftig neu zu diskutieren.

\section{ZUNEHMENDE HETEROGENITÄT DER BELEGSCHAFTEN}

Die zunehmende Heterogenität der Belegschaften in den Unternehmen und Institutionen drückt sich unter anderem in der Vielfalt der vertraglichen Beziehungen, der ethnischen Herkunft und der Altersklassen aus. Insbesondere die verschiedenen Vertragsarten sind für die Mitbestimmung ein Problem. $\mathrm{Zu}$ einem wesentlichen Instrument dürfte sich in Zukunft vor allem der „psychologische Vertrag" entwickeln.

Gebremst wird diese Entwicklung von der Tatsache, dass allmählich wieder der Wunsch nach einfachen Standardlösungen entsteht. Die Arbeitnehmer sehen sich im- (ausschließlichen) Vertretung der Arbeitnehmerinteressen bis zum anderen Extremfall des (ausschließlichen) Umgangs mit strategischen und wirtschaftlichen Problemen des Unternehmens reicht, wobei die Beteiligung an der Organisationsentwicklung und Personalpolitik eine Mittelstellung zwischen den beiden Extrempositionen einnimmt. Die Mitbestimmung sieht sich von verschiedenen Trends (derzeit) mehr oder minder in eine dieser Richtungen gedrängt.

Der erstgenannte Trend kann zwar verschiedene Formen annehmen, bleibt in seinen Konsequenzen aber doch recht eindeutig. Das Zentrum der Macht verlagert sich aus dem Unternehmen heraus, während es im Unternehmen selbst zu einer Dezentralisierung kommt. Die Ergebnisse werden nicht in erster Linie vom Trend bestimmt, sondern von den Entscheidungen des Betriebsrats, der sich auf strategischer Ebene auf die Wiederherstellung der Machtbalance, aber auch im Bündnis mit der Unternehmensleitung auf die Organisationsentwicklung und das Personalmanagement konzentrieren kann.

Auch der dritte Trend unserer Analyse, die zunehmende Vielfalt der Belegschaft, ist recht eindeutig. Er weist ebenfalls nur relativ geringe Unwägbarkeiten auf.

Die Folgen des zweiten Trends sind jedoch höchst ungewiss. Sollte es zu einer Dezentralisierung der Tarifverhandlungen kommen, stellt sich für die Betriebsräte die Frage, ob sie eine aktive Rolle in diesem Prozess spielen oder sich zurückhalten wollen. Entscheiden sie sich gegen eine aktive Rolle, bieten sich wieder die beiden Möglichkeiten des ersten Trends an. Sollte sich der Betriebsrat für eine aktive Rolle entscheiden, fallen seine Möglichkeiten jedoch höchst unterschiedlich aus: Er könnte als Partner der Gewerkschaften oder autonom von den Gewerkschaften getrennt handeln. In beiden Fällen ist es unvermeidbar, den Betriebsrat als Mitbestimmungsorgan zu erhalten, da er als Vertragspartner zur Verfügung stehen muss. Zusammenfassend lassen sich die Trends, die die Entwicklung der Mitbestimmung in die eine oder andere Richtung treiben, in vier Szenarios übersetzen (Abbildung 1). in einem Spannungsfeld bewegt, das sich aus seiner Doppelfunktion ergibt, die Arbeitnehmerinteressen $\mathrm{zu}$ vertreten und den Interessen des Unternehmens zu dienen. Aus dieser Doppelfunktion resultiert wiederum ein Spektrum möglicher Verhaltensweisen, das vom Extremfall der
3 Das Lebensverlaufprogramm trat 2006 in Kraft und bietet Beschäftigten Ansparmöglichkeiten zur Finanzierung von Zeiten unbezahlten Urlaubs, wie Bildungs-, Forschungs- oder Elternurlaub sowie für den Vorruhestand. 


\subsection{SZENARIO 1: MITBESTIMMUNG BEI DER ORGANISATIONSENTWICK- LUNG}

Die Betriebsräte multinationaler Konzerne werden infolge der Internationalisierung kaum in der Lage sein, auf die Strategie ihrer Unternehmen Einfluss zu nehmen. Auch viele kleinere Unternehmen sind so tief in Netzwerkstrukturen eingebettet, dass es für Arbeitnehmer schlichtweg unmöglich ist, strategische Entscheidungen $\mathrm{zu}$ beeinflussen.

Die dominierende Innovationsorientierung und der Einsatz vorrangig hochqualifizierter Mitarbeiter haben in den Unternehmen zu einer starken Zunahme vielfältiger direkter Partizipationsformen geführt. In einer ersten Variante könnte dies zur Folge haben, dass die betriebliche Mitbestimmung überflüssig wird, da sich die Arbeitnehmer mit den ihnen zur Verfügung stehenden Formen direkter Partizipation ohnehin Gehör verschaffen. In der zweiten Variante nutzt der Betriebsrat die einzige sich bietende Gelegenheit und macht sich zum Partner der Organisationsentwicklung.

In diesem Szenario ist die Rolle des Betriebsrats auf das Recht begrenzt, die personal- und arbeitszeitpolitischen Entscheidungen mitzutragen. Die Tarifverhandlungen bleiben den Gewerkschaften überlassen. Solange diese Aufgabenteilung genau eingehalten wird (Verhandlungen über Lohn und Arbeitsbedingungen auf der einen Seite, auf der anderen Seite Personalmanagement und Organisationsentwicklung), bleiben strittige Themen außen vor.

Liegt der Schwerpunkt auf der Organisation und dem Personalmanagement, so wird es möglich sein, Einsichten in klar umrissene Personalprobleme, aber auch Erkenntnisse über die Wandlungen im Mitbestimmungsbereich zu erlangen. Die Rolle als Partner des Personalmanagements verlangt Mut, Geschicklichkeit und Wissen.

Insgesamt könnte sich dieses Szenario in innovativen Pionierunternehmen, aber auch in bislang vertretungslosen und tariflich ungebundenen Kleinunternehmen des privaten Dienstleistungssektors, durchsetzen. Es ist zudem in Branchen und Unternehmen vorstellbar, in denen ein relativ hoher gewerkschaftlicher Organisationsgrad, ein Tarifvertrag und eine professionelle Personalpolitik herrschen.

Insbesondere $\mathrm{zu}$ den beiden letzten Konstellationen existieren empirische Hin- weise. In den letzten Jahren haben viele Unternehmen Initiativen zur Erneuerung der Mitwirkungsformen und -prozesse gestartet, darunter zum Beispiel einige große Gesellschaften wie ING, Philips und DSM sowie verschiedene öffentlich-rechtliche Institutionen. Diese Initiativen weisen einige gemeinsame Charakteristika auf: Erstens werden sie in den meisten Fällen praktisch ohne jegliche Beteiligung der Gewerkschaften in direkter Zusammenarbeit mit dem Management durchgeführt. Dies lässt sich bereits an den Namen der Projekte ablesen. Sie heißen zum Beispiel „Der Betriebsrat, ein engagierter Partner”, „Partizipation und organische Chancen" und „Mitbestimmung heißt Zusammenarbeit“. Ein weiteres gemeinsames Merkmal der Initiativen besteht darin, dass sie die Verbindung zwischen dem Betriebsrat und den Arbeitnehmern zu stärken suchen und die direkte Partizipation betonen. Ein drittes Element ist die Aufmerksamkeit, mit der man die Funktionsweise des Betriebsrats selbst beobachtet, und die Forderung, ihn in die Personalpolitik der Unternehmen zu integrieren. Die Tätigkeiten der Betriebsratsmitglieder werden als normale Arbeit betrachtet und sollen im Rahmen des Kompetenzmanagements, der Leistungsmessung und ähnlicher formaler Verfahren durchgeführt werden. Auf diese Weise sollen neue (junge) Arbeitnehmer für die Betriebsratsarbeit gewonnen werden. Diese sind zunächst davon zu überzeugen, dass eine Mitgliedschaft im Betriebsrat ihre Karriere nicht gefährdet. Weiterhin steht der Umbau der Betriebsratsstrukturen ganz oben auf der Tagesordnung. So wurden die großen zentralen (Dach-)Betriebsräte bei DSM und Philips in kleinere Kernräte umstrukturiert, die nunmehr von Projektgruppen unterstützt werden (Bruinsma/ Goodijk 2008; Smit/Vonk, im Erscheinen).

Dieses Szenario, dessen logische Folge die Verschmelzung von Mitbestimmung und Personalmanagement ist, macht ein formales Mitbestimmungsorgan unter Umständen überflüssig. Als Präzedenzfall hierfür mag der häufig ausgezeichnete Betriebsrat („Innovationspreis“) der öffentlich-rechtlichen Gesundheitsinstitution GGZ Zuid- en Midden Limburg gelten, der einen Beschluss über die eigene Auflösung gefasst hat (Praktijkblad Ondernemingsraad, September 2008).

\subsection{SZENARIO 2: MITBESTIMMUNG IN NETZWERKSTRUKTUREN}

Die Verlagerung der Kontrollinstanz auf die internationale Ebene und die Entwicklung von Netzwerkstrukturen erschweren es den Arbeitnehmervertretern, auf die Stellen und Personen Einfluss zu nehmen, die strategische Entscheidungen treffen. Schon ihre Identifizierung bereitet Probleme. In diesem Szenario provoziert die zunehmende Machtdistanz zwischen dem Zentrum der Kontrolle und den Arbeitnehmern eine Gegenreaktion. Arbeitnehmervertreter plädieren für die Demokratisierung des Entscheidungsprozesses. In großen internationalen Unternehmen kann sie in Form wechselnder Allianzen vorangetrieben werden, zum Beispiel in Kooperation mit Schlüsselorganisationen wie den europäischen Betriebsräten, der europäischen Gewerkschaftsbewegung, den nationalen Gewerkschaften, den lokalen Geschäftsleitungen, Aufsichtsräten, Aktionären wie Pensionsfonds, sozialen Organisationen etc. Auf internationaler und nationaler Ebene provozieren die Netzwerkstrukturen in Unternehmen die Bildung korrespondierender (temporärer oder permanenter) Netzwerkstrukturen in der Arbeitnehmervertretung.

In diesem Szenario bleibt das gegenwärtige System der kollektiven Tarifverhandlungen weitgehend unangetastet. Die eventuell vorhandenen Beziehungen zu den Gewerkschaften betreffen lediglich die Aufgabenteilung. Die Betriebsräte konzentrieren sich auf strategische und finanzielle Angelegenheiten sowie Fragen der Restrukturierung, die Gewerkschaften kümmern sich um Tarifverhandlungen, Arbeitsbeziehungen und Abfindungen. Wenn gemeinsame Ziele existieren, werden von Fall zu Fall Koalitionen gebildet.

Infolge seiner Beteiligung an strategischen Entscheidungsprozessen ist der Betriebsrat gezwungen, zu der Unternehmensleitung und den anderen Entscheidungszentren gute Beziehungen zu pflegen. Gleichzeitig kann durch die Betonung strategischer Fragen die Distanz zur Belegschaft steigen.

Im besten Fall können die Betriebsräte den Netzstrukturen der Unternehmen eigene Plattformstrukturen entgegensetzen und in Kooperation mit den Schlüsselorganisationen die strategischen Entscheidungsprozesse der multinationalen Unternehmen tatsächlich erfolgreich beeinflus- 
sen. Im schlimmsten Fall verstärken sich Kooperationsverweigerung aufseiten der Unternehmen und mangelnde Kenntnisse sowie fehlende Solidarität auf der Seite der Betriebsräte gegenseitig.

Empirische Belege für dieses Szenario lassen sich in den verschiedensten Bereichen finden. Bei einigen großen Restrukturierungen und Übernahmen von Finanzinvestoren verfügten die Betriebsräte über eine starke Stellung und konnten mit der Geschäftsleitung und den Gewerkschaften (beim Maschinenbauer und technischen Dienstleister Stork), institutionellen Aktionären und den Gewerkschaften (Verlagshaus PCM) bzw. nur der Geschäftsleitung (ABN AMRO) Bündnisse schmieden.

Darüber hinaus lässt sich interessanterweise feststellen, dass in jüngerer Zeit nicht nur der Gesetzgeber, sondern auch die Rechtsprechung den Arbeitnehmervertretungen zu Hilfe kam. So hat sich der Wirtschafts- und Sozialrat dafür ausgesprochen, die Anhörungsrechte des Betriebsrats in der Hauptversammlung zu stärken. Gemäß einem Gesetzentwurf vom Dezember 2007 soll der Betriebsrat darüber hinaus im Rahmen der Hauptversammlung ein Beratungsrecht im Falle der Änderung der betrieblichen Lohnpolitik erhalten. Schließlich hat die Unternehmenskammer des Berufungsgerichts Amsterdam auf Antrag der Gewerkschaften eine Untersuchung des Verlagshauses PCM angeordnet. Die Gewerkschaften machten geltend, dass der Gesellschaft seit dem Einstieg des Finanzinvestors Apax eine enorme Schuldenlast aufgebürdet worden war. Ferner beanstandeten sie einen obligatorischen Managementbeteiligungsplan, infolgedessen die Geschäftsleitung das 45-Fache ihrer ursprünglichen Investitionssumme zurückerhalten würde. Das Gericht urteilte in allen vier Punkten zugunsten der Gewerkschaften. ${ }^{4}$

\subsection{SZENARIO 3: BETRIEBSRAT ALS PARTNER DER GEWERKSCHAFTEN}

In diesem Szenario spielen die Arbeitnehmervertretungen bei der Entwicklung der tariflichen Arbeitsbedingungen eine aktive Rolle. Die Gewerkschaften führen nach wie vor die Tarifverhandlungen auf Branchenund Unternehmensebene, während die Betriebsräte die Arbeitsbedingungen auf der Ebene des Unternehmens oder der Betriebsstätte anpassen. Der Tarifvertrag erfährt also auf der Branchen- oder Konzern- ebene eine Verschlankung. Er enthält lediglich Rahmenvereinbarungen, die auf Unternehmensebene durch Vereinbarungen zwischen dem Betriebsrat und dem lokalen Arbeitgeber konkretisiert werden. Die rechtlichen Unklarheiten, die über den formalen Status der von Betriebsräten und Arbeitgebern direkt ausgehandelten Arbeitsbedingungen existierten, werden beseitigt.

In diesem Szenario entwickelt sich der Betriebsrat zu einem vollwertigen Koalitionspartner der Gewerkschaften. Zwischen den beiden Akteuren wird es zum Alltag gehören, sich in allen strukturellen Fragen zu beraten und zu koordinieren. Der Betriebsrat bekommt die volle Unterstützung der Gewerkschaften. Auf diese Weise können sich die Gewerkschaften die Betriebsräte zunutze machen und ihre eigene institutionelle Schwäche-ihre praktisch gänzliche Abwesenheit auf Ebene der Unternehmen und Betriebsstätten - überwinden.

Die Geschäftsleitung wird gegenüber dem Betriebsrat mehr und mehr die Rolle des Arbeitgebers einnehmen, der im Rahmen der tariflichen Regelung der Arbeitsbeziehungen die Interessen des Unternehmens vertritt. In seiner Doppelrolle gibt der Betriebsrat der Arbeitnehmervertretung Vorrang vor allgemeinen Unternehmensinteressen. Der Betriebsrat beteiligt sich nicht nur am Beratungsprozess, sondern nimmt auch an den Verhandlungen teil. Da jene aber nur aus Folgeverhandlungen bestehen, die sich aus den Tarifverhandlungen über die primären Arbeitsbedingungen zwischen den Gewerkschaften und Arbeitgeberverbänden auf höherer Ebene ergeben, handelt es sich hauptsächlich um ,integrative Verhandlungen” (Walton/McKersie 1965). Trotzdem können auf Unternehmens- oder Betriebsebene auch Verteilungskonflikte ausgetragen werden (distributive bargaining).

Solange integrative Verhandlungen die Situation prägen, lässt sich dieses Szenario durchaus mit einer aktiven Rolle des Betriebsrats in den Bereichen Organisationsentwicklung und Personalmanagement verbinden. Dies hätte die Integration von Tarifverhandlungen und Personalmanagement zur Folge und würde im Weiteren zu einer neuen Sozialpartnerschaft führen, in der Arbeitgeber, Gewerkschaften und Betriebsräte ein vertrauensbildendes Dreiergespann bilden und miteinander kooperieren. Sollte es zu „distributiven Verhandlungen” kommen, agieren die Betriebsräte im
Bündnis mit den Gewerkschaften in erster Linie als Arbeitnehmerräte.

Empirisch deutet nur wenig darauf hin, dass sich die Betriebsräte zu strategischen Partnern der Gewerkschaften aufschwingen könnten. Erstens hat die Dezentralisierung der Tarifverhandlungen in vielen Fällen bereits zu einer Art Mantelvereinbarung mit den Gewerkschaften geführt, die erst auf der unteren Ebene ausgestaltet wird - allerdings ohne wesentliche Beteiligung der Betriebsräte (Ten Have et al. 2007). Zweitens sind die Gewerkschaften nicht besonders daran interessiert, diese Entwicklung voranzutreiben. Sie fürchten, dass die Betriebsräte auf die Aufgaben eines Verhandlungspartners auf Betriebsebene nur unzureichend vorbereitet sind. Aber auch die Betriebsräte zögern. Einerseits spüren sie die Versuchung, die Verhandlungen über die Arbeitsbedingungen auf Betriebsebene zu führen, andererseits stellen sie den guten Willen der Arbeitgeber infrage, die sie mit hohem zeitlichen Aufwand, Schulungen und Spezialwissen in geeigneter Weise unterstützen müssten. Außerdem fürchten sie, dass die Gewerkschaften sie entweder im Regen stehen lassen oder lediglich für die undankbaren Arbeiten heranziehen werden (Smit/Vonk, im Erscheinen). Drittens ist es den meisten Arbeitgebern lieber, wenn sie Verhandlungen mit Konfliktpotenzial außerhalb der Unternehmen mit den Profis der Gewerkschaften führen können, da diese nicht ihren Belegschaften angehören. In einigen Fällen kam es deshalb sogar wieder zu einer Rezentralisierung der Verhandlungsstrukturen, z. B. beim niederländischen Bahnunternehmen Nederlandse Spoorwegen.

\subsection{SZENARIO 4: UNTERNEHMENS- GEWERKSCHAFTEN}

Dieses Szenario interpretieren wir als gravierende Schwächung der Gewerkschaftsbewegung. Der Abstand zwischen dem gewerkschaftlichen Organisationsgrad und der Reichweite der Tarifverträge nimmt hierbei in einem so großen Maße zu, dass in immer mehr Branchen die Tarifverträge auslaufen und nicht verlängert werden. Die Gewerkschaften können noch in den gröBeren Unternehmen dieser Branchen Tarifabschlüsse erzielen, in vielen Kleinunter-

\footnotetext{
4 Unternehmenskammer des Berufungsgerichts
} Amsterdam, 10. Januar 2008, LN BJ1657 
nehmen gelingt dies allerdings nicht mehr. In dieser Situation ist der Betriebsrat selbst gezwungen, über Lohn und Arbeitsbedingungen zu verhandeln, und zwar nicht als Partner der Gewerkschaften, sondern als Gewerkschaftsersatz.

Varianten dieses Szenarios reichen von der Gründung einer Firmengewerkschaft durch den Arbeitgeber (einer sogenannten gelben Gewerkschaft) über die Aushandlung der primären und/oder sekundären Arbeitsbedingungen durch den Betriebsrat bis zum Minimalszenario, in dem die Beschäftigungsbedingungen einseitig vom Arbeitgeber festgesetzt werden.

Wie im vorherigen Szenario handelt der „Unternehmer" in erster Linie als Arbeitgeber. Um die Transaktionskosten zu reduzieren, bevorzugen Arbeitgeber Vereinbarungen, die die Arbeitsbedingungen tariflich regeln. Sie wenden sich also an den Betriebsrat als Organ der Arbeitnehmervertretung. Dem Betriebsrat wird in der Folge eine Rolle aufgezwungen, die eher konfliktorische Beziehungen zum Arbeitgeber nahelegt. In diesem Szenario erscheint eine aktive Beteiligung des Betriebsrates an der Strategie- und Organisationsentwicklung wenig realistisch zu sein.

Die Möglichkeit einer „Unternehmensvereinbarung", wie sie sich im Betriebsratsgesetz von 1998 findet, hat den Weg für dieses Szenario freigemacht. Zunächst müssen in diesem Kontext jedoch noch einige rechtliche Fragen geklärt werden. Dies betrifft insbesondere den rechtlichen Status der getroffenen Vereinbarungen. Einige Zweifel an solchen Vereinbarungen können schon jetzt festgehalten werden: Auch wenn die Mitglieder eines Betriebsrats aufgrund ihrer Tätigkeit im Betriebsrat gesetzlichen Kündigungsschutz genießen, sind sie doch als Arbeitnehmer angreifbar. Darüber hinaus wird die Position des Betriebsrats als Verhandlungspartner durch das Fehlen einer Streikkasse geschwächt.

Trotz dieser ungelösten Probleme bestehen empirische Hinweise für dieses Szenario. Seine Verbreitung hängt eng mit dem gewerkschaftlichen Organisationsgrad und dem geringen Geltungsbereich der Tarifabschlüsse zusammen. Obwohl Branchenverhandlungen in den Niederlanden weit verbreitet sind, gibt es in der Tat einige Branchen ohne Branchentarifverträge. In diesen Bereichen finden sich Unternehmenstarifverträge in gewerkschaftsfreien Unternehmen, die mit den Betriebsräten auf die eine oder andere Weise über
Löhne und/oder sekundäre Arbeitsbedingungen ausgehandelt wurden. Erstens sind bei einigen in den USA oder Japan ansässigen multinationalen Gesellschaften Tarifverhandlungen mit Gewerkschaften von der Unternehmenspolitik untersagt. Dow Chemicals, ExxonMobil und Fuji wenden sich z. B. stattdessen an die Betriebsräte. In anderen Fällen haben offenbar die weitgehende Abwesenheit der Gewerkschaften (Informationstechnologie, gewerbliche Dienstleistungen) oder die geringe Machtdistanz zwischen Arbeitnehmern und Arbeitgebern (z. B. Forschungsinstitute, soziale Bewegungen wie Greenpeace, Ärzte ohne Grenzen) den Anstoß zu Verhandlungen mit dem Betriebsrat gegeben. Zweitens verhandeln in einigen anderen Fällen die Betriebsräte über die bestehenden Tarifverträge hinaus über weitere Verbesserungen der Arbeitsbedingungen. Dies ist beispielsweise in einigen der größten Unternehmen des technischen Großhandels der Fall, in denen die Betriebsräte unabhängig von den Gewerkschaften agieren (Smit/Vonk, im Erscheinen).

Bemerkenswerterweise haben die Mitglieder der Betriebsräte in diesen Fällen intensive Verbindungen $\mathrm{zu}$ den Gewerkschaftsmitgliedern, sehen sich in einer starken Position gegenüber ihrem Arbeitgeber und definieren sich gegenüber den $\mathrm{Ge}$ werkschaften nicht als Konkurrenz, sondern als Ergänzung. Zum Beispiel wurde in der Chemiebranche eine Plattform für diejenigen Betriebsräte ins Leben gerufen, die aus Unternehmen mit Unternehmenstarifverträgen stammen oder die Arbeitsbedingungen selbst verhandeln. Die Plattform dient hauptsächlich dem Vergleich der Arbeitsbedingungen, und ihre Versammlungen werden auch von Gewerkschaftsfunktionären besucht, die die Plattform beraten (ebd.).

\section{Schlussfolgerungen}

Wir haben vier Szenarios über die Zukunft der Mitbestimmung in den Niederlanden entworfen, die gleichzeitig eintreten können. Es scheint sogar möglich, dass sich verschiedene Szenarios in einer einzigen Organisation parallel ausbilden. Zum Beispiel ist es durchaus vorstellbar, dass sich eine Form der Arbeitnehmervertretung durchsetzt, die als Partner des Personalma- nagements und der Gewerkschaften gleichermaßen agiert (Szenarios 1 und 3). Das vierte Szenario (Mitbestimmung im Rahmen einer Unternehmensgewerkschaft) lässt sich wohl am wenigsten mit den anderen Szenarios koppeln, da Verhandlungen über Lohn und andere Beschäftigungsbedingungen (ein Nullsummenspiel) und die Einflussnahme auf strategischer Ebene oder eine reibungslose Zusammenarbeit in Personalfragen nicht vereinbar scheinen.

Die nach wie vor lückenhaften empirischen Belege deuten an, dass Szenario 1 (Mitbestimmung in der Organisationsentwicklung) an Dynamik gewinnt. In Anbetracht von Szenario 2 (Mitbestimmung in Netzwerkstrukturen) haben die Betriebsräte - vielleicht etwas unerwartet - Rückendeckung vom Gesetzgeber und von der Rechtsprechung erhalten. Es bleibt jedoch abzuwarten, welche Auswirkungen dies auf die Funktionsweise der Arbeitnehmervertretung haben wird. Zugleich bestehen keine Anzeichen, dass Szenario 3 eintritt (Betriebsräte als Partner der Gewerkschaften). Die Gewerkschaften scheinen das Interesse an den Betriebsräten verloren zu haben. Aus der Dezentralisierung der Tarifverhandlungen scheint in erster Linie die Individualisierung der Arbeitsbedingungen $\mathrm{zu}$ folgen, was die Kompetenzen der Betriebsräte als Organ der Arbeitnehmervertretung übersteigt. Szenario 4 (Entstehung einer Unternehmensgewerkschaft) scheint sich als reales Szenario in gewerkschaftlich nicht organisierten Unternehmen und Institutionen bereits auszubreiten, insbesondere dort, wo die Ausdehnung des Geltungsbereichs der Tarifverträge (derzeit $82 \%)$ stark rückläufig ist.

Da die Tradition einvernehmlicher Arbeitgeber-Arbeitnehmer-Beziehungen in den Niederlanden sehr stark ist (Stichwort: „Poldermodell“), sind drastische Veränderungen auf kurze Sicht nicht zu erwarten. Doch können sie aufgrund des stetig abnehmenden gewerkschaftlichen Organisationsgrads auch nicht ausgeschlossen werden. Fest steht, dass sich zwischen den Branchen Unterschiede ergeben haben, die zum Teil auf die Praxis der industriellen Beziehungen in den Unternehmen aus den USA und Japan zurückgehen. Die Globalisierung wird die Ausdifferenzierung weiter verstärken, und da sich die niederländische Volkswirtschaft für eine Fragmentierung ihrer Arbeitsbeziehungen geöffnet hat, muss mit einer weiteren Ausdifferenzierung der Mitbestimmungspraxis gerechnet werden. 


\section{LITERATUR}

Becker, H. (1994): Werken met scenario's, Amsterdam Bruinsma, G./Goodijk, R. (Hrsg.) (2008): OR Strategie en Beleid. Het huishouden van de OR, Alphen a/d Rijn

De Schutter, O./Deakin, S. (2005): Introduction: Reflexive Governance and the Dilemma's of Social Regulation, in: De Schutter, O./Deakin, S. (Hrsg.): Social Rights and Market Forces, Brussels, S. 1-27

Marsden, D. (2004): The "Network Economy" and Models of the Employment Contract, in: British Journal of Industrial Relations 4, S. 659 684

Nonaka, I./Takeuchi, H. (1995): The Knowledge Creating Company, New York

Schilstra, K./Smit, E. (2005): Voeten op de vloer. Strategische keuzes in de belangenbehartiging van werknemers, Amsterdam
Schwartz, P. (1991): The art of the long view, London

Smit, E./Vonk, L. (im Erscheinen): Vier scenarios in de praktijk, Utrecht Ten Have, C./Oei, P./Kraan, K. (2007): Arbeidsvoorwaarden en arbeidsverhoudingen op ondernemingsniveau - AVON Monitor, Hoofddorp Van het Kaar, R. (2007): De Nederlandse medezeggenschap in Europees perspectief, Amsterdam

Van het Kaar, R./Smit, E. (Hrsg.) (2007): Vier scenario's voor de toekomst van de medezeggenschap, Delft

Visser, J. (2006): Union membership statistics in 24 countries, in: Monthly Labor Review 1, S. 38-49

Walton, R./McKersie, R. (1965): A Behavioral Theory of Labor Negotiations, New York 